\title{
Vestibular therapy improved motor planning, attention, and balance in children with attention deficit hyperactivity disorders: a randomized controlled trial
}

\author{
Sedigheh Farokhi Moghadam ${ }^{1}$, Hojjat Allah Haghgoo ${ }^{1 *}$, Ebrahim Pishyareh ${ }^{1}$, Enayatollah Bakhshi ${ }^{2}$, Nima Rezazadeh $^{3}$, Reza Rostami $^{4}$ and \\ Vahid Sadeghi ${ }^{5}$ \\ ${ }^{1}$ Department of Occupational Therapy, School of Rehabilitation, University of Social Welfare and Rehabilitation Sciences, Tehran, Iran \\ ${ }^{2}$ Department of Biostatistics, University of Social Welfare and Rehabilitation Sciences, Tehran, Iran \\ ${ }^{3}$ Department of Audiology, School of Rehabilitation, University of Social Welfare and Rehabilitation Sciences, Tehran, Iran \\ ${ }^{4}$ Department of Psychology, Faculty of Psychology and Educational Sciences, University of Tehran, Tehran, Iran \\ ${ }^{5}$ Department of Psychology, Faculty of Education \& Psychology, Shahid Beheshti University Tehran, Iran
}

\begin{abstract}
Introduction: Considering the crucial role of the vestibular system in motor and cognitive functions.

Methodology: To investigate the effects of vestibular therapy on the motor and cognitive improvements, 63 children with attention deficit hyperactivity disorders were assessed for eligibility. A total of 38 participants (19 female and 19 male), with 7-12 years of age, were recruited and randomized to receive vestibular therapy referred as an experimental group. Moreover, another group of participants, referred to as control group, received no intervention for the same time period. Intervention implemented for two months, 3 sessions per a week, with each session lasting for 30 minutes. All the subjects were evaluated using the the Bruininks-Oseretsky Test of Motor Proficiency, Integrated Visual and Auditory Continuous Performance Test, and the Cambridge Neuropsychological Test Automated Battery before and after intervention and data were analyzed using analysis of covariance.
\end{abstract}

Results: Vestibular therapy improved motor performances $(\mathrm{P}<0.006)$, response control $(\mathrm{P}<0.016)$, auditory $(\mathrm{P}<0.012)$ and visual $(\mathrm{P}<0.012)$ attention, and motor planning $(\mathrm{p}<0.02)$ in the experimental group.

Conclusion: Vestibular stimulation was effective in improving motor skills, response inhibition, attention, and motor planning in children with attention deficit hyperactivity disorders.

\section{Introduction}

Children with attention-deficit/hyperactivity disorder (ADHD) have a continuous pattern of severe and frequent inattention and/or hyperactivity, restlessness, and failure in academic achievement $[1,2]$. Some researchers suggested that the inappropriate use of cognitive strategies to solve problems and regulate behaviors is associated with attention deficit [3-6]. Behavioral dysregulation, as a difficulty in inhibiting maladaptive behaviors such as hyperactivity, impulsivity inattention, and aggression) $[7,8]$, about equal to the impairment in executive function $[9,10]$. Executive function is an umbrella term that is used to explain cognitive processes underlying functions such as motor planning, memory, attention, problem-solving, reasoning, response inhibition, cognitive flexibility, multi-tasking, initiating, organization, and monitoring the functions [11]. Researchers believe that some components of executive functions are insufficient in people with ADHD. Especially, these children have more difficulties in attention span, sustained attention, response inhibition, organization, working memory, and motor planning $[12,13]$.

About $47 \%$ to $69 \%$ of these children have motor disorders [14] including problems in gross and fine motor skills, motor coordination, and motor control, which are mostly related to attention deficit severity and ADHD symptoms [15-18]. Furthermore, accompanying with motor control problems, these children also show significant balance disorders. Almost, about $30 \%$ to $50 \%$ of these children suffer from poor balance and low coordination [19]. Our previous research showed that about $60 \%$ of children with a combined type of ADHD suffer from vestibular dysfunction [20,21].

Motor and postural control disorders are explainable with sensory processing dysfunction especially in relation to vestibular system [22]. Vestibular system deficiency manifests itself as an imbalance in postural control muscles' activation, which causes an inappropriate postural condition. This postural imbalance will lead to severe cognitive problems such as problems in paying attention to the task, which is more common in children with ADHD [23]. According to sensory processing approach, many disruptive behaviors in ADHD children are compensatory reactions to stimulate the vestibular system [24]. In this approach, neural systems involved in processing and integrating

${ }^{\star}$ Correspondence to: Hojjat Allah Haghgoo, Department of Occupational Therapy, University of Social welfare and Rehabilitation Sciences, Tehran, Iran, E-mail: h.haghgoo@yahoo.com

Key words: vestibular therapy, cognition. attention, motor planning, ADHD

Received: April 21, 2018; Accepted: April 28, 2018; Published: April 30, 2018 

controlled trial

sensory modalities control both executive functions and motor behaviors such that to provide a substrate for the adaptive behaviors [16]. The sensory modalities include (but not limited to) visual, auditory, tactile, olfactory, kinesthetic, proprioceptive, and balance or vestibular senses. When the neural system is unable to process and integrate certain information from the sensory modalities, it results in sensory processing dysfunction [18]. Therefore, sensory information may receive naturally, but affect motor and cognitive performances in an abnormal way [15]. Approximately, $16 \%$ of the normal populations have symptoms of sensory processing disorder, while the prevalence of these symptoms in children with ADHD is within 40-84\% [25-27]. One class of the sensory processing disorder subtypes is sensory-basedmotor disorders including disturbances in body posture (problems with balance and core stability) and dyspraxia (problems in planning and sequencing of movements) $[15,24]$.

Considering the dysfunction of the vestibular system in ADHD $[20,21]$, some researchers reported the effects of vestibular stimulation on hyperactivity symptoms in these children $[28,29]$. In these reports, the researchers focused mainly on hyperactivity symptoms, while motor problems including balance, muscle strength, and fine and gross motors are not addressed. Furthermore, the impact of interventions on the subclasses of executive function, as the underlying cause of ADHD, has not been investigated.

Regarding the vital role of sensory processing as the basis for the executive function, the prominent role of vestibular system in sensory processing [25], and the powerful effects of vestibular stimulation on mechanisms underlying motor control [30], we proposed that therapeutic exercises with an emphasis on vestibular stimulation to improve the motor and cognitive performances in the children with ADHD. Overall, we investigated the effects of vestibular stimulation on the motor and cognitive functions in children with ADHD.

\section{Materials and method}

This study was approved by the ethical committee of the University of Social Welfare and Rehabilitation Sciences, Tehran, Iran, ethics' code USWR.AC.IR.1392.114. Moreover, a written informed consent was signed by all participants' caregivers (parents) before the study. A total of 63 children with ADHD aged 7-12 years old were recruited from the pediatric outpatient clinic of XXX Rehabilitation Center, XXX. They were diagnosed as children with ADHD based on psychiatrist interview according to DSM-IV-TR.

All the subjects were referred to an audiologist and occupational therapist to assess their vestibular function. Having at least two positive tests from the below-mentioned assessments were considered as inclusion eligibility. Assessment tools for inclusion criteria included the Wechsler Intelligence Scale for children (WISC-V) - to confirm normal IQ (minimal IQ 90), Vestibular Evoked Myogenic Potential - Rotary chair testing, and Oculomotor Tests [20]. The existence of significant medical or neurological disorders (such as depression, anxiety, learning disorder, epilepsy, deafness, and visual problems) was considered as exclusion criteria.

Of 63 assessed subjects, 38 participants (19 female and 19 male), recruited and randomized using numbered containers to receive vestibular therapy during academic education (19 subjects, $M=10$ and $\mathrm{F}=9$ ) were referred as the experimental group while those with academic education ( 19 subjects, $M=9, F=10$ ) was referred to the control group.

All subjects in the experimental group participated in a comprehensive Vestibular Rehabilitation Therapy (VRT) program [31] containing overall balance and gate, postural stability, and eye movement exercises $[31,32]$. Exercises were modified in consideration of the age of the subjects and their balance abilities and attention capacities to enhance gaze stability, postural stability, and daily living activities. The rehabilitative intervention was conducted by an occupational therapist research assistant for two months, 3 sessions per week, and each session lasting for 30 minutes, while the subjects in the control group participated only in their usual academic education. They received the same VRT program for two months after post-assessment as an ethical consideration.

Before and after the intervention period, all the subjects were assessed using the Bruininks-Oseretsky Test of Motor Proficiency (BOTMP), IVA-plus, and CANTAB (Stock of Cambridge or SOC subtest) to evaluate the effects of the intervention on motor and cognition functions.

\section{Intervention protocol}

(Vestibular Rehabilitation Therapy) was developed and approved by rehabilitation professionals including occupational therapy and audiology faculty members according to the vestibular sensory stimuli, which stimulate the semicircular channels, utricle, and saccule organs [32-36]. This protocol includes vestibular exercises for central and peripheral vestibular structures. Intervention protocol was done by an occupational therapist. General vestibular exercises were applied for all subjects in the experimental group in the first halftime of each session; however, in the second half, exercises were tailored to each participant according to vestibular disorders diagnosed during balance and vestibular assessments. These exercises targeted mainly on either peripheral or central vestibular disorders [36].

Subjects in both groups were evaluated using the BOTMP, Integrated Visual and Auditory Continuous Performance Test, and Cambridge Neuropsychological Test Automated Battery before and after the intervention. All assessments were done by examiners blind to subject's assigned group.

\section{Assessment tools}

The Wechsler Intelligence Scale for children Fifth Edition (WISC-V): The test provides five primary index scores (i.e., Verbal Comprehension Index, Visual Spatial Index, Fluid Reasoning Index, Working Memory Index, and Processing Speed Index). This test was developed by David Wechsler in New York [37]. The test-retest reliability of WISC-V has been reported in the range of $44 \%$ to $94 \%$ and split-half reliability coefficients of subtests were from $43 \%$ to $94 \%$ [38]. Testing was conducted by a trained clinical psychologist to generate a full-scale IQ.

Bruininks-Oseretsky Test of Motor Proficiency (BOTMP): Researchers use this assessment battery to collect a comprehensive index of fine and gross motor skills for children aged 4.5 to 14.5 years old. This tool consists of 8 subscales. Four subtests evaluate gross motor skills (running speed and agility, balance, bilateral coordination, and power), 3 subtests assess fine motor skills (response speed, visual-motor control, and upper limbs speed and dexterity), and a subtest assesses both gross and fine motor titled upper limb coordination [39].

\section{CANTAB test}

CANTAB test is composed of a series of neuropsychological tests. This tool was developed at the University of Cambridge computer to assess the cognitive components especially those related to the frontal and temporal areas of the brain. The battery consists of 22 neuropsychological tests in the cognitive domains, such as motor skills, 

controlled trial

visual attention, visual/spatial memory, working memory, decisionmaking, response control, executive function, and attention. All tests are non-verbal and their validity has been evaluated by various studies [36]. In this study, we analyze two subtests; the Motor Screening test (MOT) to screen the subjects' eligibility for test and Stockings of Cambridge (SOC) to assess subjects' executive function.

The Motor Screening test (MOT): The MOT is applied to calm the subject and familiarize him/her with computer and touch screen in a play-form activity. First, the subject learns to point by the index finger on a cross appears on the screen (i.e., to touch it). The test simultaneously screens visual problems, movement disorder, and comprehension, and determines whether students follow simple instructions or not. If the $\mathrm{Z}$ score is between +1 and -1 , the person is able to be assessed by CANTAB test; otherwise, the child has auditory or visual problems [40]. In fact, the outcomes of this measure are the speed of response and the accuracy of pointing. It is noteworthy that all subjects were able to follow instructions and their $\mathrm{Z}$ score was in the acceptable range.

The Stocking of Cambridge (SOC) was originally introduced as a test of executive function. This test requires the ability to plan spatial and strategic motor programs and measures the frontal lobe's function. In this test, the screen is divided into upper and lower halves by a horizontal line. In the upper half, three columns with different numbers of circles of different colors appear in a special pattern. In the bottom half, different numbers of colored circles are placed in three columns in a pattern different from the pattern of the upper half. To run the test, the subject should move colored circles in three columns of the lower half of the screen to match the pattern on the upper half and reconstruct the upper half. Fewer numbers of movements to complete the pattern indicates the higher ability of subject in motor programming. The program reports average time to think before acting and the smallest amount number of movements to run the test as scores on this subtest. The difficulty of levels increases by reaching the smallest amount of movementss needed to complete the work reaches five movements to two movements. The time spent to reconstruct the pattern and number of movementss required will consider as a measure of the first programming ability of subject $[40,41]$.

Integrated Visual and Auditory Continuous Performance Test (IVA + Plus): The IVA + Plus is a test of attention that consists of two stimuli modalities; i.e., visual and auditory. This test was designed to measure two important domains: attention and impulse control. This test does not make a diagnosis but helps the examiner to make a diagnosis. The primary use of this tool is to help the diagnosis of Attention DeficitHyperactivity Disorder; however, it can also be applied to evaluate other attention disorders, and problems with self-control related to head injury, sleep disorders, depression, anxiety, learning disabilities, dementia, and other health problems. The tool measures the response to 500 intermixed auditory and visual stimuli spaced 1.5 seconds apart. The program presents stimuli in a random order so that the subject is not able to predict the next stimulus. The test is applicable for children from 6 years and older and adults. Testing along with instructions and training takes about 20 minutes. The task is composed of response or non-response (response inhibition) to 500 visual or auditory stimuli. The subject sees visual stimuli on the screen and hears auditory stimuli by headphone. The display time for each visual stimulus is $167 \mathrm{MS}$ and for each auditory stimulus is 500 MS. Here, the task is to click the mouse when the stimulus is either an auditory or a visual "number 1 " and to refrain from clicking when the stimulus is an auditory or a visual "number 2 ". The variables reported by this test are the number of correct answers, the number of non-responding to (omitting) the target stimulus, the number of responding to non-target stimuli (commission error), and reaction times in the milliseconds. IVA + Plus test is used to evaluate the inhibition of response after a period of consecutive responses. So, it needs a sustained and continuous attention. The psychometric results indicate a sufficient sensitivity for the test as $92 \%$ and $89 \%$ to correct diagnosis of ADHD in children. Furthermore, testretest reliability for different parts of the test is reported to be $59 \%$ to $93 \%[42,43]$.

Intervention protocol was developed and approved by rehabilitation professionals including occupational therapy and audiology faculty members according to the vestibular sensory stimuli, which stimulate the semi-circular channels, utricle and saccule organs. This protocol includes vestibular exercises for central and peripheral vestibular structures used for experiment group [32,34,35]. These training exercises include overall balance exercises such as jumping on trampolines, lying and sitting on the therapy ball, maintaining balance standing, sitting, squatting, etc. on a tilt board, taking different positions like standing, kneeling and crawling on rotating boards or scooters, walking on the balance beam, linear and rotational movements back and forth, left and right on a normal swing, passing over the obstacle, rolling, simple and difficult moves backward [34], stability exercises like standing on one leg with eyes open and closed [32], standing with feet in tandem, moving back and forth, staring and standing while changing legs width, exercise with head lights, head spin, rotating the headtrunk, turning heads while walking, practicing on the ramp, going up and down stairs, saccadic eye movements using eye gaze stability, simultaneous vestibular and somatosensory inputs [33], simultaneous use of visual and vestibular inputs, using all sensory information for postural control, and using all senses to control the body position.

Data were analyzed using SPSS version 20 software. Leven test and t-test were used to examine the consistency of data. KolmogorovSmirnov test was used to evaluate the normal distribution of data. According to the results, with respect to the normal distribution of data, parametric tests (analysis of covariance) were used to compare the impact of interventions on changes in the two groups. The significance level for all tests was 0.05 .

\section{Results}

\section{Study population}

A consolidated Standards of Reporting Trials (CONSORT) flow chart.

Totally, 63 children with ADHD were assessed for eligibility. Twenty-five subjects were excluded because of not meeting inclusion criteria. Thirty-eight participants (19 females and 19 males) were randomized to receive vestibular therapy and education (19 subjects, $\mathrm{M}=10, \mathrm{~F}=9$ ) referred as an experimental group and receive only education (19 subjects, $M=9, F=10$ ) referred as a control group. All the participants completed the post-test evaluations.

The mean of ages was an $8.95 \pm 1.68$ year in experimental group and $8.63 \pm 1.46$ year in the control group with no significant differences in age or gender. The results of Leven Test for equality of variances and T-Test for equality of mean BOTMP's scores showed no differences between the two groups before intervention.

Then, the normal distribution of test scores of BOTMP was measured in both groups using Kolmogorov-Smirnov test; and the results indicated that all data were distributed normally $(\mathrm{P}>0.05)$. The impact of the intervention on the BOTMP' scores in participants. 

controlled trial

As can be seen in vestibular rehabilitation therapy resulted in a significant improvement in balance $(\mathrm{P}>0.001)$, and also in the total gross motor abilities in the experimental group compared to the control group $(P=0.006)$. A subset analysis of total gross motor skills showed that while running speed and agility $(\mathrm{P}=0.89)$, bilateral coordination $(\mathrm{P}=0.41)$, and strength $(\mathrm{P}=0.99)$ did not show significant change in the intervention group compared to control group, a significant improvement in balance resulted in significant improvement in the total gross motor abilities $(\mathrm{P}=0.006)$. Furthermore, the analysis of individual subtests showed that visual motor control was improved significantly in the intervention group when compared to the control group $(\mathrm{P}=0.007)$ while the subtest of total fine motor skills revealed no significant difference in the two groups $(\mathrm{P}=0.123)$. In addition, the total score of BOTMP has changed significantly in the intervention group in comparison with the control group $(\mathrm{P}=0.006)$.

According to vestibular rehabilitation program resulted in significant improvements in response control $(\mathrm{p}=0.016)$, visual vigilance $(\mathrm{p}=0.012)$, and auditory vigilance $(\mathrm{p}=0.012)$ in the experimental group compared to the control group. Furthermore, the effects of the intervention on motor planning are presented.

According, vestibular stimulation resulted in significant changes in the SOC scores in the experimental group compared to the control group $(\mathrm{p}=0.02)$. In contrast, the mean initial thinking time after the intervention (latency) showed no significant changes between the two groups after intervention $(\mathrm{p}=0.72)$.

\section{Discussion}

In the present study, children with a combined type of ADHD received vestibular stimulation for two months. The intervention improved significantly their gross motor skills, balance, and visualmotor control in comparison with the subjects in the control group. Furthermore, the intervention resulted in significant increases in response control and visual vigilance while a significant reduction in auditory vigilance (a marker of inattention) [44]. Finally, CANTAB evaluation results showed that vestibular therapy resulted in a better function of motor planning. However, in the present study, there was no significant change in fine motor, running speed and agility, bilateral coordination, and strength in the intervention group compared to the control group.

\section{Vestibular system and motor function}

Acquiring motor coordination, development of balance, movement in space, visual-motor integration, and visual-motor coordination are all dependent on the normal function of the vestibular system [45]. The vestibular inputs activate vestibular nuclei in the brainstem, and these nuclei are projected to basal ganglia via posterior intralaminar nuclei and effect on cortical regions such as premotor cortex and parietal cortex [46]. Furthermore, there are connections between vestibular system and cerebellum in one side and with different brain cortices as well as connections between the vestibular system and other parts of the central nervous system including the thalamus, which explains the effects of vestibular stimulation on executive function and motor development skills [47]. Previous studies have shown that caloric vestibular stimulation activates the cerebellar outputs to the prefrontal and visual cortex [46]. In addition, vestibular stimulation in children with cerebral palsy resulted in the significantly improved development of gross motor skills and motor coordination [44]. Furthermore, the effects of vestibular stimulation and sensory-motor activities resulted in calming and hyperactivity control in children with ADHD and the improvement in gross motor skills [48]. Different brain structures including cortex, right inferior frontal regions, the left sensorimotor cortex, basal ganglia, cerebellum and bilateral vermis, right cingulate cortex, and brainstem on both sides are involved in the etiology of ADHD [49,50]. It could be implied that dysfunction in these areas can result in poor postural control (hypotonia, or hypertonia, poor control of limbs, and balance), problem in motor learning (learning new skills, motor planning, coping with changes, and automation), and poor sensory-motor coordination (coordination between/within limbs, the sequencing of motor activities, using feedback to correct motion, timing, predicting and planning strategies). Therefore, it may be concluded that vestibular stimulation resulted in modulating vestibular inputs to widespread brain structures involved in motor control and improved motor activities in the studied children.

Finally, according to CANTAB results, vestibular therapy resulted in significant improvement in motor planning abilities. Motor planning or praxis is the brain's ability to receive, organize, and perform unfamiliar activities [51]. In motor planning disorder, affected person loses the ability to carry out new activities, even though motor and perceptual capacities are intact. Vestibular system and its projections to cerebellum play an integral role in motor planning, and any vestibular disorder can cause defects in motor planning [51]. Therefore, it seems that the vestibular system plays a major role in motor planning and motor control systems. This effect could be either by vestibular effects on consciousness system, and increasing attention, decreasing impulsivity and modulating feedback loops, or in an interaction with cerebellum and proprioceptive inputs.

\section{Vestibular system and Cognitive function}

One of the main reasons for impulsivity in children with ADHD is their problems in controlling response to sensory stimuli, which result in impulsivity and hyperactivity [52,53]. It is assumed that some ADHD symptoms are due to reticular formation dysfunction. This system processes information received from sensory pathways through widespread connections [54] and change the level of consciousness and arousal by the effect on the projection of sensory information to the thalamus and from there to the cortex. Accordingly, another theory that arises in this context relates to the hypothesis that intra-laminar nuclei of the thalamus are involved in integrating the inputs associated with the level of consciousness, attention, working memory, control of eye movements and gaze, and are modulated by vestibular stimulation [46]. It seems that this reticular activating system is modified by modulatory sensory stimuli. Connections between vestibular systems with reticular formation involve in regulating consciousness levels and selective attention $[45,55]$. On the other hand, vestibule-cerebellar connections, and vestibular projections to different brain areas including the thalamus may explain the relationship between vestibular inputs and behavioral regulation [47]

In our previous study, a sensory integration program with an emphasis on proprioceptive and vestibular senses resulted in a decrease in symptoms of ADHD [29]. In accord with our findings, rotational vestibular stimulation improved significantly the scores of hyperkinetic children in Connors questionnaire and a significant reduction in the symptoms of Attention-Deficit/Hyperactivity [28]. Researchers also reported that hyperactivity behaviors were reduced significantly after semicircular canals stimulation [56]. It seems that vestibular stimulation changes the level of consciousness and probably, modulate consciousness system which resulted in orienting the child's attention to the environmental stimuli, affects correct response to stimuli, and prevents impulsivity. 

controlled trial

In order to control coordinated fine and rapid movements, sensory information from somatosensory, visual and vestibular systems must be integrated to interpret complex sensory environments and provide the information necessary to control and coordinate these movements. The sensory information from the somatosensory, vestibular, and visual systems are integrated. Moreover, the relative weight applied to each of these inputs depends on the goals of motor tasks and environmental contexts. As the sensory environment of individuals changes, the relative dependence on each sensory system must be re-measured. For example, healthy people rely on somatosensory (70\%), vision (10\%), and vestibular (20\%) information in an appropriate illuminated environment with a stable support basis [21].

The control of quick and fine movements is a complex skill that takes place based on the interaction of dynamic sensory-motor processes and includes many basic physiological systems that can be influenced by pathology or clinical constraints. Damage to any of the subsystems creates various disruptions correlated with the affected special structures. Effective rehabilitation of balance, coordination, and control of gross or fine movements require a better understanding of multiple mechanisms of motor control and the provision of pathologically-based interventions for each subsystem and consideration of total systemdamages. Therefore, many systems must be evaluated and intervened.

At the end, it worth mentioning that only one care provider (an occupational therapist) offered therapeutic services in this research for all subjects, and she was blind to the research aims. She was qualified for application of vestibular therapy protocol and tailoring the program for each subject, individually. Secondly, the tester was blind to each subject's allocation group. And the comparator, also, was blind to allocated groups; he only tested differences between the subjects in the two groups, encoded by 1 and 2. Finally, there are some debates around the relationship between subjects and therapist that may have specific therapeutic effects. Therefore, this therapeutic relationship might overestimate the treatment effects.

\section{Conclusion}

Vestibular system operates inappropriately in children with ADHD. Vestibular stimulation can improve balance, gross motor skills, and visual motor control. Furthermore, these stimuli decrease impulsivity and hyperactivity via regulating consciousness and attention levels and improve motor planning and execution. It seems that while some problematic behaviors in children with ADHD may be modified by a behavioral modification, but interventions based on neurological conditions such as vestibular therapy are more efficient in addressing these behavioral problems. Therefore, the vestibular stimulant can be suggested as a therapeutic intervention to help in functional improvements of children with ADHD.

\section{Impact}

Attention, Motor dexterities, Motor planning abilities, and Balance in in children with ADHD improved by Vestibular stimulation, while Impulsivity in these subjects are decreased. Considering neurodevelopmental basis of the disorder, this therapeutic program can improve physical and cognitive abilities in psychological conditions such as ADHD and provide a neural substrate to facilitate normal development.

\section{Acknowledgements}

The author(s) declare(s) no potential conflicts of interest with respect to the research, authorship, and/or publication of this article.
The author(s) received no financial support for the research, authorship, and/or publication of this article. I/we appreciate helps of the subjects, their families, co-workers, teachers, school administrators, and staff, without whose help this research was impossible.

\section{References}

1. Bebko GM, Franconeri SL, Ochsner KN, Chiao JY (2014) Attentional deployment is not necessary for successful emotion regulation via cognitive reappraisal or expressive suppression. Emotion 14: 504-512. [Crossref]

2. Barkley RA (1997) ADHD and the Nature of Self-control: Guilford Press, USA.

3. Bertocci MA, Bebko G, Olino T, Fournier J, Hinze AK, et al. (2014) Behavioral and emotional dysregulation trajectories marked by prefrontal-amygdala function in symptomatic youth. Psychol Med 44: 2603-2615. [Crossref]

4. Castellanos FX, Aoki Y (2016) Intrinsic functional connectivity in attention-deficit/ hyperactivity disorder: A science in development. Biol Psychiatry Cogn Neurosci Neuroimaging 1: 253-261. [Crossref]

5. Reardon SN, Naglieri JA (1992) PAAS cognitive processing characteristics of normal and ADHD males. J School Psychol 30: 151-163.

6. Romo L, Legauffre C, Guilleux A, Valleur M, Magalon D, et al. (2016) Cognitive distortions and ADHD in pathological gambling: A national longitudinal case-control cohort study. J Behav Addict 5: 649-657. [Crossref]

7. Martin CS, Earleywine M, Blackson TC, Vanyukov MM, Moss HB, et al. (1994) Aggressivity, inattention, hyperactivity, and impulsivity in boys at high and low risk for substance abuse. J Abnorm Child Psychol 22: 177-203. [Crossref]

8. Mezzich AC, Giancola PR, Tarter RE, Lu S, Parks SM, et al. (1997) Violence, suicidality, and alcohol/drug use involvement in adolescent females with a psychoactive substance use disorder and controls. Alcohol Clin Exp Res 21: 1300-1307. [Crossref]

9. Pennington BF, Ozonoff S (1996) Executive functions and developmental psychopathology. J Child Psychol Psychiatry 37: 51-87. [Crossref]

10. Sepeta LN, Casaletto KB, Terwilliger V, Facella-Ervolini J, Sady M, et al. (2017) The role of executive functioning in memory performance in pediatric focal epilepsy. Epilepsia 58: 300-310. [Crossref]

11. Sonuga-Barke EJ, Sergeant JA, Nigg J, Willcutt E (2008) Executive dysfunction and delay aversion in attention deficit hyperactivity disorder: nosologic and diagnostic implication. Child Adolesc Psychiatr Clin N Am 17: 367-384. [Crossref]

12. Barkley RA (2006) Attention deficit- Hyperactivity disorder: A handbook for diagnosis and treatment. New York: The Guilford Press, USA.

13. Walshaw PD, Alloy LB, Sabb FW (2010) Executive function in pediatric bipolar disorder and attention-deficit hyperactivity disorder: in search of distinct phenotypic profiles. Neuropsychology Rev 20: 103-120. [Crossref]

14. Fliers E, Rommelse N, Vermeulen SH, Altink M, Buschgens CJ, et al. (2008) Moto coordination problems in children and adolescents with ADHD rated by parents and teachers: effects of age and gender. J Neural Transm (Vienna) 115: 211-220. [Crossref]

15. Buderath P, Gartner K, Frings M, Christiansen H, Schoch B, et al. (2009) Postural and gait performance in children with attention deficit/hyperactivity disorder. Gait Posture 29: 249-254. [Crossref]

16. Pitcher TM, Piek JP, Hay DA (2003) Fine and grossmotor ability in maleswith ADHD Dev Med Child Neurol 45: 525-535. [Crossref]

17. Shum SB, Pang MY (2009) Children with attention deficit hyperactivity disorder have impaired balance function: involvement of somatosensory, visual, and vestibular systems. J Pediatr 155: 245-249. [Crossref]

18. Tseng MH, Henderson A, Chow SM, Yao G (2004) Relationship between motor proficiency, attention, impulse, andactivity in children with ADHD. Dev Med Child Neurol 46: 381-388. [Crossref]

19. Cohen ME (1995) Occupation and visual/vestibular interaction in vestibular rehabilitation. Otolaryngol Head Neck Surg 112: 526-532. [Crossref]

20. Lotfi Y, Rezazadeh N, Moossavi A, Haghgoo HA, Rostami R, et al. (2017) Preliminary evidence of improved cognitive performance following vestibular rehabilitation in children with combined ADHD (cADHD) and concurrent vestibular impairment. Auris Nasus Larynx 44: 700-707. [Crossref]

21. Peterka R (2002) Sensorimotor integration in human postural control. J Neurophys 88: 1097-1118. [Crossref] 
Moghadam SF (2018) Vestibular therapy improved motor planning, attention, and balance in children with attention deficit hyperactivity disorders: a randomized controlled trial

22. Hunter MC, Hoffman MA (2001) Postural control: visual and cognitive manipulations. Gait Posture 13: 41-48. [Crossref]

23. Vyas RM, Alperovich M, Grayson BH, McCarthy JG, Rodriquez ED (2015) From multidisciplinary to interdisciplinary to transdisciplinary care: an evolution in craniofacial surgery. Plast Reconstr Surg 135: 796e-797e. [Crossref]

24. Miller LJ, Nielsen DM, Schoen SA, Brett-Green BA (2009) Perspectives on sensory processing disorder: a call for translational research. Front Integr Neurosci 3: 22. [Crossref]

25. Ben-Sasson A, Carter AS, Briggs-Gowan MJ (2009) Sensory over-responsivity in elementary school: prevalence and social-emotional correlates. J Abnorm Child Psychol 37: 705-716. [Crossref]

26. Dunn W, Bennett D (2002) Patterns of sensory processing in children with attention deficit hyperactivity disorder. Occup Ther J Res 22: 4-15.

27. Mulligan S (1996) An analysis of score patterns of children with attention disorders on the Sensory Integration and Praxis Tests. Am J Occup Ther 50: 647-654. [Crossref]

28. Clark DL, Arnold LE, Crowl L, Bozzolo H, Peruggia M, et al. (2008) Vestibular Stimulation for ADHD: randomized controlled trial of Comprehensive Motion Apparatus. J Atten Disord 11: 599-611. [Crossref]

29. Ebrahimi M, Abas MW, Haghgoo HA, Masoumeh PT, Fatemeh D (2013) The effectiveness of sensory-motor integration with an emphasis on proprioceptive and vestibular senses on the symptoms of Attention Deficit/ Hyperactivity Disorder (ADHD). J Res Rehabil Sci 9: 220-231.

30. Bundy AE, Murray EA, Lane SJ (2002) Sensory integration theory and practice. Philadelphia: FA Davis Co, USA.

31. Lotfi Y, Rezazadeh N, Moossavi A, Haghgoo H, Moghadam SF, Pishyareh E, et al. (2016). Introduction of pediatric balance therapy in children with vestibular dysfunction: Review of indications, mechanisms, and key Exercises. Iran Rehabil J 14: 5-14.

32. Han BI, Song HS, Kim JS (2011) Vestibular rehabilitation therapy: review of indications, mechanisms, and key exercises. J Clin Neurol 7: 184-196. [Crossref]

33. Gill-Body KM, Krebs DE, Parker SW, Riley PO (1994) Physical therapy management of peripheral vestibular dysfunction: two clinical case reports. Phys Ther 74: 129-142. [Crossref]

34. Herdman S, Whitney HS (2007) Vestibular Rehabilitation. Interventions for the patien with vestibular hypofunction. Philadelphia: FA Davis Co, USA.

35. Keim RJ, Cook M, Martini D (1992) Balance rehabilitation therapy. Laryngoscope 102: 1302-1307. [Crossref]

36. Luciana M, Nelson CA (2002) Assessment of neuropsychological function through use of the Cambridge Neuropsychological Testing Automated Battery: performance in 4- to 12-year-old children. Dev Neuropsychol 22: 595-624. [Crossref]

37. Kaplan RM, Saccuzzo DP (2008) Psychological Testing: Principles, Applications, and Issues (7th Edition ed.): Wadsworth, USA.

38. Nazari S, Bohluli B, Varedi P, Besharatizade R (2014) Carpentering in septorhinoplasty: a novel technique to straighten the deviated nasal septum. J Oral Maxillofac Surg 72: 157-163. [Crossref]
39. Bruininks R, Bruininks B (2005) Minneapolis, MN: NCS Pearson.

40. Torgersen J, Flaatten H, Engelsen BA, Gramstad A (2012) Clinical Validation of Cambridge Neuropsychological Test Automated Battery in a Norwegian Epilepsy Population. J Behav Brain Sci 2: 108-116.

41. Limited CC (2012) CANTABeclipseTM Test Administration Guide Manual version 5.0.0.

42. Arble E, Kuentzel J, Barnett D (2014) Convergent validity of the Integrated Visual and Auditory Continuous Performance Test (IVA+Plus): associations with working memory, processing speed, and behavioral ratings. Arch Clin Neuropsychol 29: 300312. [Crossref]

43. Riccio CA, Reynolds CR, Lowe P, Moore JJ (2002) The continuous performance test: a window on the neural substrates for attention? Arch Clin Neuropsychol 17: 235-272. [Crossref]

44. Chee FK, Kreutzberg JR, Clark DL (1978) Semicircular canal stimulation in cerebral palsied children. Phys Ther 58: 1071-1075. [Crossref]

45. Beginnings G (2010) The vestibular system: treating attention deficit disorder (www. gr8beginnings.com)

46. Schiff ND, Pulver M (1999) Does vestibular stimulation activate thalamocortica mechanisms that reintegrate impaired cortical regions? Proc Biol Sci 266: 421-423. [Crossref]

47. Arendt RE, MacLean WE Jr, Halpern LF, Youngquist GA, Baumeister AA (1991) The influence of rotary vestibular stimulation upon motor development of nonhandicapped and Down syndrome infants. Res Dev Disabil 12: 333-348. [Crossref]

48. Niklasson M, Niklasson I, Norlander T (2009) Sensorimotor therapy: using stereotypic movements and vestibular stimulation to increase sensorimotor proficiency of children with attentional and motor difficulties. Percept Mot Skills 108: 643-669. [Crossref]

49. Niedermeyer E, Naidu SB (1997) Attention-deficit hyperactivity disorder (ADHD) and frontal-motor cortex disconnection. Clin Electroencephalogr 28: 130-136. [Crossref]

50. Zang YF, He Y, Zhu CZ, Cao QJ, Sui MQ, et al. (2007) Altered baseline brain activity in children with ADHD revealed by resting-state functional MRI. Brain Dev 29: 83-91. [Crossref]

51. Clark DL, Kreutzberg JR, Chee FK (1977) Vestibular stimulation influence on motor development in infants. Science 196: 1228-1229. [Crossref]

52. Neal MV (1969) The relationship between a regimen of vestibular stimulation and developmental behavior of the small premature infant. Nurs Res Conf 5: 43-57.

53. Neal MV (1977) Vestibular stimulation and development of the small premature infant Commun Nurs Res 8: 291-302. [Crossref]

54. Baumeister AA, Henderson K, Pow JL, Advokat C (2012) The early history of the neuroscience of attention-deficit/hyperactivity disorder. J Hist Neurosci 21: 263-279. [Crossref]

55. Wilhelmsen J (1996) The effects of vestibular stimulation and cuing on attention in an autistic child, in occupational therapy (www.spdfoundation.net).

56. Christine S (1994) Case study of the effects of vestibular stimulation on reading skil in children with learning disability and occompanying vestibular dysfunction, in occupational therapy.

Copyright: (C2018 Moghadam SF. This is an open-access article distributed under the terms of the Creative Commons Attribution License, which permits unrestricted use, distribution, and reproduction in any medium, provided the original author and source are credited. 